\title{
Evaluation of Airway Wall Thickness Via High-Resolution Computed Tomography in Mild Intermittent Asthma
}

\author{
Selvi Asker MD, Muntecep Asker MD, and Bulent Ozbay MD
}

\begin{abstract}
INTRODUCTION: This study aims to evaluate bronchial thickness via thorax high-resolution computed tomography (HRCT) in subjects with mild intermittent asthma in comparison with healthy control subjects. METHODS: A total of 37 out-patients (mean \pm SD age $=36.7$ years $(9.7$ years); $54.8 \%$ males) with mild intermittent asthma and 13 healthy control subjects (mean \pm SD age $=25.0$ years $(2.9$ years $)$; $61.5 \%$ males) were included in this case control study. Data on demographics, pulmonary function test results, and segmental and subsegmental thorax HRCT results were recorded. The ratio of bronchial wall thickness to bronchial lumen diameter (T/D) and bronchial wall area percentage (WA\%) were calculated for all cases. RESULTS: Subject and control groups were similar in terms of pulmonary function test results, and total and subsegmental T/D values. Mean \pm SD subsegmental WA $\%$ values at the level of inferior pulmonary vein $(55.6 \%$ [16.8\%] vs $41.7 \%[7.4 \%], P=.047)$ and $2 \mathrm{~cm}$ above the diaphragm $(49.8 \%$ [15.8\%] vs $38.6 \%$ $[10.4 \%], P=.046)$ were significantly higher in subjects than control subjects. No significant correlation of overall and subsegmental T/D and WA\% values to age in both groups, and to asthma duration in subjects. CONCLUSION: Our findings revealed an increase in bronchial wall thickness in peripheral airways in subjects with mild intermittent asthma regardless of the duration of asthma. This may indicate a need to also administer anti-inflammatory or bronchodilator therapy, which is effective in peripheral airways during the early period of the disease. Key words: asthma; mild intermittent asthma; thorax high-resolution computed tomography; respiratory function tests; bronchial wall thickening. [Respir Care 2014;59(4):550-556. () 2014 Daedalus Enterprises]
\end{abstract}

\section{Introduction}

Asthma results in irreversible air-flow obstruction in a subgroup of individuals via mechanisms proposed to include remodeling in the airway, but that are as yet unclear, ${ }^{1}$ which leads to considerable morbidity and accounts for a high percentage of the health costs of asthma in these subjects. $^{2}$

Dr Selvi Asker is affiliated with the Department of Chest Diseases, and Dr Muntecep Asker is affiliated with the Department of Cardiology, Van Higher Education Training and Research Hospital, Van, Turkey. Dr Ozbay is affiliated with the Department of Chest Diseases, Mugla University Faculty of Medicine, Mugla, Turkey.

The authors have disclosed a relationship with Chiesi Turkey. The authors have disclosed no other conflicts of interest.

Correspondence: Dr Selvi Asker, Van Yüksek İhtisas Hastanesi, 65710 Van, Turkey. E-mail: selviasker@mynet.com.

DOI: $10.4187 /$ respcare. 02780
Characterized by an increase in the thickness of the airway wall, which results from mucosal infiltration by inflammatory cells; deposition of connective tissue on the extracellular matrix; and an increase in muscle mass, mucus glands, and vessel area, ${ }^{3}$ remodeling of the airway wall in subjects with asthma has been demonstrated to occur not only in the central airways, but also in the peripheral small airways. ${ }^{4}$ The remodeling of small airways, in particular, may be largely responsible for irreversible air-flow obstruction and an increase in airway responsiveness. ${ }^{5}$ It is therefore important to assess the structural changes in the airway when deciding on the most appropriate asthma therapy for individual asthmatic patients. ${ }^{5}$

Remodeling is routinely evaluated based on certain parameters including internal and external diameters, the area of bronchial cross section or bronchial lumen, and their derivative indexes. ${ }^{6}$ The use of the dynamics of these parameters in making diagnostic decisions has been considered to necessitate the use of measurement techniques enabling precise and reproducible assessment of the bronchial 
tree components. ${ }^{6}$ Recently, owing to improved resolution that enables the identification of features $100-200 \mu \mathrm{m}$ in size and allows for assessment of small airways in the region of 1.5-2 mm diameter, ${ }^{7}$ high-resolution computed tomography (HRCT) has gained increasing interest in this context. HCRT has been advocated for assessing the structural changes in the asthmatic lung in relation to the severity of the disease and clinical parameters. ${ }^{2,5}$

The present study was designed to evaluate bronchial thickness via thorax HRCT in subjects with mild intermittent asthma receiving as-needed short-acting $\beta_{2}$ agonists but not inhaled corticosteroids in comparison with healthy control subjects. Additionally, the relation of bronchial thickness to age, pulmonary function test results, and asthma duration was evaluated.

\section{Methods}

\section{Study Population}

A total of 50 participants, including 37 out-patients $($ mean \pm SD age $=36.7$ years $(9.7$ years $) ; 54.8 \%$ males $)$ with mild intermittent asthma and 13 healthy control subjects (mean $\pm \mathrm{SD}$ age $=25.0$ years $(2.9$ years); $61.5 \%$ males), were included consecutively in this case control study. All patients had previously received diagnoses of asthma based on spirometry and methacholine challenge test results at chest diseases out-patient clinics with no exacerbation within the past 6 months; therefore, patients were currently classified as having mild intermittent asthma. Consequently, a repeat methacholine inhalation test was not considered to be necessary.

Female or male out-patients $\geq 18$ years of age, in whom mild intermittent asthma had been diagnosed (i.e., daytime symptoms less than twice per week, nocturnal symptoms less than twice per month, feeling completely normal during the symptom-free periods, and daily peak expiratory flow [PEF] variability of $<20 \%$ ), who had not experienced exacerbations within the last 6 months, were receiving no asthma-related treatment other than short-acting $\beta_{2}$ agonist inhaler therapy, were nonsmokers, did not have a history of regular treatment for another disease (inactive lung tuberculosis, bronchiectasis, lung malignancy, heart failure, and diabetes mellitus), and had no history of upper respiratory airway infection within the last 6 weeks were included in the present study. The control group was composed of healthy nonsmoker subjects without a history of respiratory or allergic disease, upper respiratory airway infection within the last 6 weeks, and regular treatment for another disease. Patients not meeting the inclusion criteria; patients with a known exposure to environmental pollutants such as motor exhaust, smoke, pesticides, solvents, and molds from wet agricultural environments; patients with obstructive sleep apnea (OSA) based on the presence

\section{QUICK LOOK}

\section{Current knowledge}

Asthma results in an increase in the thickness of the airway wall. Remodeling of the airway wall in asthma has been demonstrated to occur in small airways, which are largely responsible for irreversible air-flow obstruction and an increase in airway responsiveness. Highresolution computed tomography has been advocated for assessing the structural changes in the asthmatic lung in relation to severity of the disease.

\section{What this paper contributes to our knowledge}

In subjects with mild intermittent asthma, there was an increase in bronchial wall thickness in peripheral airways regardless of the duration of asthma. This finding may indicate the need for early anti-inflammatory or bronchodilator therapy.

of nighttime snoring, and witnessed apnea along with identification of scores of $>10$ on the Epworth Sleepiness Scale; and patients who were confirmed to have additional cardiac pathologies were excluded from the study.

Asthma control levels were classified as controlled, partly controlled, and uncontrolled, based on daytime symptoms, limitations of activities, nocturnal symptoms/awakening, need for reliever/rescue treatment, lung function, and the number of exacerbations, while asthma attack severity was categorized into four groups (mild, moderate, severe, and life-threatening) based on the Global Initiative for Asthma (2007 revision) guidelines. ${ }^{8}$ In relation to replacement of the concept of disease severity with disease control in updated asthma guidelines, ${ }^{9}$ the subject population in the present study was determined to be composed of subjects with controlled asthma with as-needed use of short-acting $\beta_{2}$ agonist inhaler therapy.

Written informed consent was obtained from each subject following a detailed explanation of the objectives and protocol of the study, which was conducted in accordance with the ethical principles stated in the Declaration of Helsinki and approved by the institutional ethics committee.

\section{Data Collection}

Demographic (age, gender) and clinical features (duration of asthma, smoking status, and history of medical treatment) of subjects were recorded. Pulmonary function tests and thorax HRCT scans were performed on the same day as the discontinuation of agonist therapy, 12 or $24 \mathrm{~h}$ prior to measurements in all cases, as well as thorax HRCT 

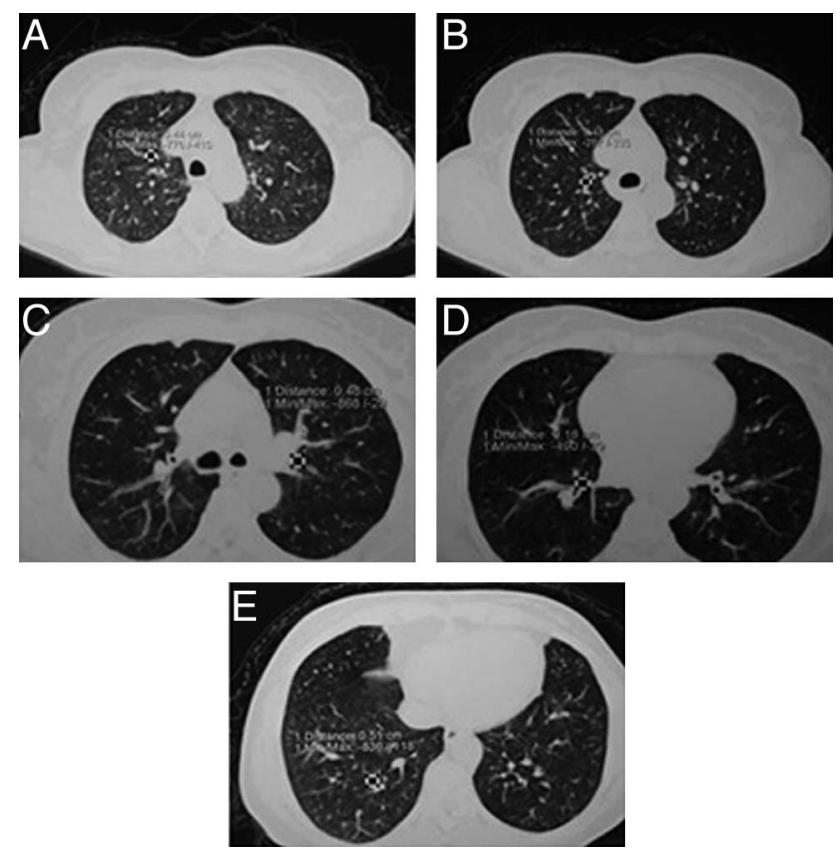

Fig. 1. Subsegmental high-resolution $C T$ sections taken at $A$. superior margin of aortic arch B. $1 \mathrm{~cm}$ above the carina C. carina D. level of inferior pulmonary vein E. $2 \mathrm{~cm}$ above the diaphragm.

slices that involved the segmental and subsegmental airways of the lung. The ratio of the bronchial wall thickness to the bronchial lumen diameter (T/D) and bronchial wall area percentage (WA\%) were calculated for all cases.

\section{HRCT Measurements}

HRCT measurements were performed by the same experienced radiologist in all cases using a multidetectorrow computed tomography system called Sensation 4 (Siemens, Erlangen, Germany) at a $120 \mathrm{kV}$ peak and $90 \mathrm{~mA}$ with scan collimation of $1.0 \mathrm{~mm}$ and section thickness of $2.0 \mathrm{~mm}$. After the initial evaluation of sections, bronchial thickness and bronchial diameter were measured at 5 different levels of both the segmental and subsegmental layers. The window width and level used in the assessment of wall thickness were based on characteristics dictated by results of the validation studies conducted with phantom models and cadaveric lungs. ${ }^{10,11} \mathrm{HRCT}$ sections were taken from the superior margin of the aortic arch, $1 \mathrm{~cm}$ above the carina, the carina, at the level of the inferior pulmonary vein, and $2 \mathrm{~cm}$ above the diaphragm (Fig. 1). Given the statement on the influence of oblique sections on bronchial wall thickness, ${ }^{12}$ the ratio of the long to the short diameter of the bronchus being assessed was determined to be $<1.5$. To overcome the probability of individual variability in measurements, certain ratios were calculated including (1) bronchial wall thickness, expressed as a ratio to the total airway diameter (T/D) and calculated using the formula of

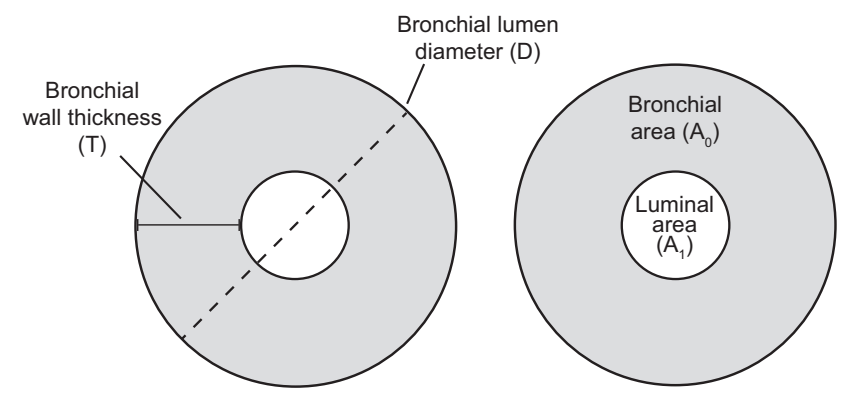

Fig. 2. Left - calculation of bronchial wall thickness ratio (T/D). Right - calculation of bronchial wall area percentage $\left(A_{0}-A_{1} / A_{0}\right)$.

bronchial wall thickness/bronchial lumen diameter; and (2) bronchial wall area, expressed as WA\% using the formula of bronchial area - luminal area/bronchial area (Fig. 2).

\section{Spirometric Measurements}

Spirometric measurements were made via the Vitalograph Alpha (serial No. AL 12907) device to evaluate $\mathrm{FEV}_{1}, \mathrm{FEV}_{1} / \mathrm{FVC}$, and PEF parameters.

\section{Statistical Analysis}

Statistical analysis was performed using SPSS version 13.0 (SPSS Inc. Chicago, Illinois). The Student $t$ test was used for the comparison of T/D and WA\% values between the subject and control groups. The relation of pulmonary function test results to demographics and asthma duration was evaluated using Pearson correlation analysis. Data were expressed as the mean $\pm \mathrm{SD}$ and percentage, where appropriate. $P<.05$ was considered statistically significant.

\section{Results}

Asthma subjects were significantly older (mean $\pm \mathrm{SD}$ age $=36.7$ years [9.7 years] vs 25.0 years [2.9 years], $P<.001)$ compared with control subjects, but there was no significant difference regarding gender distribution (males 54.8 vs $61.5 \%, P<.640$ ). The mean $\pm \mathrm{SD}$ asthma duration was 42.7 months (6.0 months) in subjects with mild intermittent asthma (Table 1).

There was no gender influence on the total mean $\pm \mathrm{SD}$ T/D (0.32 [0.08] in males vs 0.30 [0.07] in females) and WA\% values $(47.4 \%$ [9.6\%] in males vs $48.4 \%$ [8.7\%] in females).

\section{Bronchial Wall Thickness Ratio in Subject Versus Control Groups}

The subject and control groups were similar in terms of total thickness as well as thickness measured from sections 
Table 1. Comparison of Subject and Control Groups in Terms of Study parameters

\begin{tabular}{|c|c|c|c|}
\hline Study parameters & $\begin{array}{l}\text { Asthmatic } \\
\text { subjects } \\
(n=37)\end{array}$ & $\begin{array}{l}\text { Control } \\
\text { subjects } \\
(n=13)\end{array}$ & $P$ \\
\hline Male gender, $n(\%)$ & $20(54.8)$ & $8(61.5)$ & .64 \\
\hline Age (years) & $36.7(9.7)$ & $25.0(2.9)$ & $<.001$ \\
\hline Duration of asthma, months & $42.7(6.0)$ & & \\
\hline \multicolumn{4}{|l|}{$\mathrm{T} / \mathrm{D}$} \\
\hline $\begin{array}{l}\text { At the superior margin of } \\
\text { aortic arch }\end{array}$ & $0.30(0.11)$ & $0.35(0.06)$ & .06 \\
\hline At $1 \mathrm{~cm}$ above the carina & $0.29(0.12)$ & $0.35(0.07)$ & .07 \\
\hline At the carina & $0.30(0.15)$ & $0.36(0.06)$ & .15 \\
\hline $\begin{array}{l}\text { At the level of inferior } \\
\text { pulmonary vein }\end{array}$ & $0.29(0.15)$ & $0.33(0.07)$ & .21 \\
\hline At $2 \mathrm{~cm}$ above the diaphragm & $0.31(0.14)$ & $0.38(0.11)$ & .06 \\
\hline Total thickness & $0.30(0.09)$ & $0.36(0.06)$ & .47 \\
\hline \multicolumn{4}{|l|}{ WA\% } \\
\hline $\begin{array}{l}\text { At the superior margin of } \\
\text { aortic arch }\end{array}$ & $48.0(17.1)$ & $41.9(6.6)$ & .15 \\
\hline At $1 \mathrm{~cm}$ above the carina & $47.5(12.9)$ & $40.5(9.1)$ & .05 \\
\hline At the carina & $53.7(16.7)$ & $47.3(16.7)$ & .08 \\
\hline $\begin{array}{l}\text { At the level of inferior } \\
\text { pulmonary vein }\end{array}$ & $55.6(16.8)$ & $41.7(7.4)$ & .05 \\
\hline At $2 \mathrm{~cm}$ above the diaphragm & $49.8(15.8)$ & $38.6(10.4)$ & .05 \\
\hline Total area & $50.9(10.0)$ & $42.0(7.0)$ & .004 \\
\hline \multicolumn{4}{|l|}{ Respiratory function tests } \\
\hline $\mathrm{FEV}_{1}, \%$ predicted & $86.0(7.8)$ & $93.1(10.9)$ & .12 \\
\hline $\mathrm{FEV}_{1} / \mathrm{FVC}$ & $84.5(6.1)$ & $88.8(4.8)$ & .25 \\
\hline $\mathrm{PEF}, \mathrm{L} / \mathrm{min}$ & $85.5(1.5)$ & $90.4(5.4)$ & .11 \\
\hline \multicolumn{4}{|c|}{$\begin{array}{l}\text { Values are given as the mean }=\mathrm{SD} \text { unless stated otherwise. } \\
\mathrm{T} / \mathrm{D} \text { ratio }=\text { bronchial wall thickness to bronchial lumen diameter ratio } \\
\text { WA\% } \% \text { bronchial wall area percentage } \\
\text { PEF = peak expiratory flow }\end{array}$} \\
\hline
\end{tabular}

taken from the superior margin of aortic arch, $1 \mathrm{~cm}$ above the carina, the carina, at the level of inferior pulmonary vein, and $2 \mathrm{~cm}$ above the diaphragm (see Table 1).

\section{Bronchial Wall Area Percentage in Subject Versus Control Groups}

Besides overall mean \pm SDWA $\%$ values (50.9\% [10.0\%] vs $42.0 \%$ [7.0\%], $P<.004$ ), subsegmental WA\% values for the level of inferior pulmonary vein $(55.6 \%$ [16.8\%] vs 41.7\% [7.4\%], $P=.047$ ) and $2 \mathrm{~cm}$ above the diaphragm (49.8\% [15.8\%] vs $38.6 \%$ [10.4\%], $P=.046)$ were also significantly higher in the subject group compared with control group (see Table 1).

\section{Respiratory Function Tests in Subject Versus Control Groups}

Subject and control groups were similar in terms of mean $\pm \mathrm{SD} \mathrm{FEV}_{1}, \mathrm{FEV}_{1} / \mathrm{FVC}$, and PEF values (see Table $1)$.

\section{Correlation of T/D and WA\% to Age, Respiratory Function Test Results, and Asthma Duration}

In both asthmatic and control subjects, there was no significant correlation of overall and subsegmental T/D and WA\% values to age, asthma duration, and respiratory function test results.

\section{Discussion}

Our findings related to the evaluation of T/D and WA\% values in subjects with mild intermittent asthma in comparison to healthy control subjects revealed no difference between subjects and control subjects in terms of segmental and subsegmental T/D, whereas there were significantly higher total segmental and subsegmental WA\% values, including at the level of the inferior pulmonary vein and $2 \mathrm{~cm}$ above the diaphragm, in subjects than in control subjects. In both groups, age, asthma duration, and pulmonary function test results were not correlated to T/D and WA\% values.

In recent years, HRCT has become available in the clinical practice as a noninvasive method enabling objective evaluation of lung parenchyma and bronchial wall thickening in parallel to asthma severity, 2,13,14 with no interobserver difference indicating the reproducibility of the method. ${ }^{2}$

Supporting the concept of chronic inflammation causing bronchial wall thickening and remodeling, the main findings in the literature suggest that subjects with asthma have greater airway wall thickening than healthy subjects, and that those with severe disease have thicker airways than subjects with milder disease. ${ }^{15}$

In the present study, airway dimensions of both segmental and subsegmental bronchi were measured directly from magnified HRCT images, and the results were expressed based on calculation of the T/D and the WA\%. While similar studies in the literature evaluated bronchial wall thickness based on the measurement of T/D only (Boulet et $\mathrm{al}^{16}$ ) and the calculation of WA\% only (Okazawa et $\mathrm{al}^{17}$ ), we used both T/D and WA\% to assess thickening of the segmental and subsegmental bronchi, and compared our results to those in studies conducted by Awadh et al, ${ }^{13}$ Niimi et al, ${ }^{18}$ and Gono et al..$^{5}$ The results of these studies revealed inconsistent data, including no bronchial wall thickening in large airways, ${ }^{16}$ and increased thickness in large airways, ${ }^{18}$ in airways $<6 \mathrm{~mm}$ in luminal diameter, ${ }^{17}$ and also in both large and small airways ${ }^{13}$ among asthmatic subjects compared to control subjects, as well as in asthmatic subjects with deficient reversible airflow obstruction compared to asthmatic subjects with normal spirometric functions. ${ }^{5}$

However, given the higher degree of airway thickening in subjects with nearly fatal and moderate asthma than in 
subjects with mild asthma, ${ }^{13}$ the inclusion of control subjects with mild intermittent asthma in the present study seems consistent with our findings of similar T/D values for both segmental and subsegmental airways between the subject and control groups.

Accordingly, significantly higher subsegmental WA\% values for the measurements at the level of inferior pulmonary vein and $2 \mathrm{~cm}$ above the diaphragm in the subject group than in the control group in the present study seem to indicate that increased bronchial wall thickness among subjects with mild intermittent asthma was specific to small peripheral airways, as has also been confirmed in a study on childhood asthma. ${ }^{19}$

Besides, increased segmental and subsegmental bronchial wall area in our subjects compared to control subjects supports the idea that the use of WA\% in the evaluation of both segmental and subsegmental bronchial walls was associated with increased bronchial wall thickness in both small and large airways in subjects with mild-tomoderate asthma ${ }^{13,17}$ and in subjects with nearly fatal asthma, with the thickening being directly proportional to the severity of the disease. ${ }^{13}$ Likewise, given that the inflammation involves the whole wall of the bronchus, leading to an obstruction, measurements taken from a single point were reported to be associated with incorrect findings, while WA\% was indicated to be a more relevant method in the reflection of the changes in bronchial wall thickness. ${ }^{20}$

The pathological features of bronchial wall thickening may reflect not only irreversible airway remodeling, such as hypertrophy of mucus-secreting glands, subepithelial fibrosis, marked thickening of basement membrane, hyperplasia, and hypertrophy of airway smooth muscle, but also reversible components, such as edema, infiltration by inflammatory cells, and bronchoconstriction. ${ }^{5}$ Hence, it has been recommended that asthma be optimally controlled before HRCT assessment to exclude the influence of the potentially reversible airway changes that can occur in a radiological study of airway structural changes. ${ }^{2,21}$ In our study, a population composed of subjects with mild intermittent asthma that was under control underwent HCRT, performed by the same experienced radiologist, to measure both the segmental and subsegmental airways. Our findings revealed that subjects and control subjects were similar in terms of total and subsegmental bronchial wall thickness, while significantly higher bronchial wall area was determined in terms of total segmental and subsegmental regions, consistent with the predominant involvement of peripheral small airways in subjects than in control subjects.

Likewise, Gono et $\mathrm{al}^{5}$ evaluated the irreversible structural changes of airways with the aid of HRCT scanning via the inclusion of subjects with stable and asymptomatic asthma as well as elimination of the reversible compo- nents. They concluded that asthmatic subjects with incomplete reversibility of air-flow obstruction showed an increased airway wall thickness on HRCT scans compared with asthmatic subjects with complete reversibility of airflow obstruction, as in other studies. 22,23

Accordingly, lack of a significant difference between our subjects with mild intermittent controlled asthma and healthy control subjects in terms of T/D and respiratory function parameters is consistent with the significant correlation between wall thickness and air-flow obstruction, and the similarity of asymptomatic asthmatic subjects without air-flow obstruction to healthy subjects, as reported by Gono et al. ${ }^{5}$

Data on the correlations of wall thickness and area to lung function are inconsistent and potentially compromised due to the lack of elimination of reversible changes of airways. ${ }^{18}$ Hence, the lack of any correlation between respiratory function parameters and segmental T/D and WA\% values in the present study is in line with past studies indicating that airway wall thickening was not correlated with air-flow obstruction. ${ }^{16,22,24,25}$ However, this finding is unlike the statement that thickening induces air-flow obstruction in subjects with asthma, which was based on reported significant and negative correlation between indices of airway wall thickness and $\mathrm{FEV}_{1}$ percent predicted. ${ }^{12}$

The carina was the only subsegmental region in our study where the bronchial thickness showed a significant correlation with $\mathrm{FEV}_{1} / \mathrm{FVC}$ in terms of T/D in subjects, and in terms of both T/D and WA\% in control subjects. However, there was no marked increase in either bronchial wall thickness or area in this subsegmental area compared to control subjects. Hence, our findings seem to support that thickening of the airway wall assessed by HRCT scanning seems not to be correlated with air-flow obstruction and that small airway function may be influenced more by bronchial wall thickening than are respiratory function parameters. ${ }^{2,22}$

In fact, in a study concerning bronchoarterial ratio and bronchial wall thickness seen on HRCT scans in asymptomatic subjects, the bronchial/arterial ratio was reported to be influenced by aging, with the HRCT-analyzed bronchial/arterial ratio being significantly higher in patients $>65$ years of age. Hence, it was stated that when this ratio is used for the quantitative analysis of pulmonary and cardiovascular disease, the influence of age should be considered. ${ }^{26}$

However, although the subject and control groups in the present study were not homogeneous in terms of age and gender distribution, there was no significant relation of bronchial wall thickness measurements to age and gender both in the subject and control groups. Hence, our findings seem to be free of the possible compromising influences of age and gender disparity. Nevertheless, it is obvious that 
these findings should be justified by future larger-scale studies to be confident of this statement. Besides, asthma duration had no significant influence on bronchial wall thickness and area, as was the case with the effect of asthma duration on bronchial thickness, as reported by Chetta et $\mathrm{al}^{27}$ in subjects with mild persistent and persistent asthma.

Given the significant alterations in bronchial wall thickness associated with smoking, ${ }^{26}$ exposure to environmental pollutants, ${ }^{28}$ and the presence of OSA, ${ }^{29}$ it is worth noting that our study population of subjects with mild asthma was composed of nonsmoker individuals without OSA and a history of exposure to environmental pollutants.

The identification of bronchial wall thickening and an increased cross-sectional area particular to peripheral airways in the present study seems to support the idea that even subjects with mild asthma had thickening of the airway wall compared with healthy control subjects. ${ }^{13}$ Besides, given that subjects with mild asthma had a long history of asthma despite no admission to the hospital and normal baseline lung function test results, it was hypothesized that chronic inflammatory changes lead to airway wall thickening even in subjects with mild disease. Hence, although inhaled corticosteroids could not be administered to our subjects, since, according to the guidelines, the subjects did not meet the criteria for taking inhaled corticosteroids, as shown in previous studies ${ }^{30-32}$ the possibility of prevention of the development of chronic airway obstruction with the early use of inhaled corticosteroids seems notable and has been emphasized in some national guidelines. ${ }^{13,32}$

However, it must be emphasized that the difference between subjects with mild asthma and healthy subjects in terms of airway wall thickening has been reported to be small with unknown clinical importance. ${ }^{13}$ A considerable overlap between asthmatic and healthy subjects was indicated to support the view that this aspect of airway remodeling does not necessarily discriminate individual asthmatic subjects from healthy subjects, whereas airway remodeling is generally present in asthmatic subjects as a group, which may be related to physiological change and can be positively affected by long-term therapy with inhaled corticosteroids. ${ }^{25,33}$

A major limitation of our study is the small sample size, which limits the generalizability of our findings by making them arguable in terms of statistical power, whereas the study inclusion criteria are in accordance with the good control of external effects, and international guidelines were well followed. Hence, our study has merit and is relevant from a clinical point of view as it suggests that even mild intermittent asthma may induce structural changes in the small airways.
In conclusion, our findings revealed significant increases in bronchial wall thickness considering peripheral airways in subjects with mild intermittent asthma regardless of the duration of asthma. To be justified via future randomized controlled trials, this may indicate a need to administer anti-inflammatory or bronchodilator agents as effective therapy for peripheral airways also in the early period of disease. In addition, our findings confirm that HCRT scanning is a suitable noninvasive method for the measurement of bronchial wall thickness.

\section{ACKNOWLEDGMENTS}

We thank Chiesi Turkey for their support.

\section{REFERENCES}

1. Brown PJ, Greville HW, Finucane KE. Asthma and irreversible airflow obstruction. Thorax 1984;39(2):131-136.

2. Little SA, Sproule MW, Cowan MD, Macleod KJ, Robertson M, Love JG, et al. High resolution computed tomographic assessment of airway wall thickness in chronic asthma: reproducibility and relationship with lung function and severity. Thorax 2002;57(3):247-253.

3. Bousquet J, Chanez P, Lacoste JY, White R, Vic P, Godard P, Michel FB. Asthma: a disease remodeling the airways. Allergy 1992; 47(1):3-11.

4. Kuwano K, Bosken CH, Pare PD, Bai TR, Wiggs BR, Hogg JC. Small airway dimensions in asthma and in chronic obstructive pulmonary disease. Am Rev Respir Dis 1993;148(5):1220-1225.

5. Gono H, Fujimoto K, Kawakami S, Kubo K. Evaluation of airway wall thickness and air trapping by HRCT in asymptomatic asthma. Eur Respir J 2003;22(6):965-971.

6. Mincewicz G, Rumiński J, Krzykowski G. Application of adjusted subpixel method (ASM) in HRCT measurements of the bronchi in bronchial asthma subjects and healthy individuals. Eur J Radiol 2012; 81(2):379-383.

7. King GG, Müller NL, Paré PD. Evaluation of airways in obstructive pulmonary disease using high-resolution computed tomography. Am J Respir Crit Care Med 1999;159(3):992-1004.

8. Global Initiative for Asthma (GINA). Global strategy for asthma management and prevention. Revised 2007. http://www.ginasthma. org/. Accessed 1/28/14.

9. Global Initiative for Asthma (GINA). GIobal strategy for asthma managemement and prevention. Revised 2012. http://www.ginasth ma.org/. Accessed 1/28/14.

10. Brown RH, Herold CA, Hirshman EA, Zerhouni EA, Mitzner W. In vivo measurements of airway reactivity using high resolution computed tomography. Am Rev Respir Dis 1991;144(1):208-212.

11. McNamara AE, Müller NL, Okazawa M, Arntorp J, Wiggs BR, Paré PD. Airway narrowing in excised canine lungs measured by high resolution computed tomography. J Appl Physiol 1992;73(1):307316.

12. Kasahara K, Shiba K, Ozawa T, Okuda K, Adachi M. Correlation between the bronchial subepithelial layer and whole airway wall thickness in subjects with asthma. Thorax 2002;57(3):242-246.

13. Awadh N, Müller NL, Park CS, Abboud RT, FitzGerald JM. Airway wall thickness in subjects with near fatal asthma and control groups: assessment with high resolution computed tomographic scanning. Thorax 1998;53(4):248-253.

14. Park CS, Müller NL, Worthy SA, Kim JS, Awadh N, Fitzgerald M. Airway obstruction in asthmatic and healthy individuals: inspiratory 


\section{Evaluation of Airway Wall Thickness Via HRCT in Mild Intermittent Asthma}

and expiratory thin-section CT findings. Radiology 1997;203(2):361367.

15. Mclean AN, Sproule MW, Cowan MD, Thomson NC. High resolution computed tomography in asthma. Thorax 1998;53(4):308-314.

16. Boulet L-P, Bélanger M, Carrier G. Airway responsiveness and bronchial-wall thickness in asthma with or without fixed airflow obstruction. Am J Respir Crit Care Med 1995;152(3):865-871.

17. Okazawa M, Müller N, McNamara AE, Child S, Verburgt L, Paré PD. Human airway narrowing measured using high resolution computed tomography. Am J Respir Crit Care Med 1996;154(5):1557-1562.

18. Niimi A, Matsumoto H, Amitani R, Nakano Y, Mishima M, Minakuchi M, et al. Airway wall thickness in asthma assessed by computed tomography: relation to clinical indices. Am J Respir Crit Care Med 2000;162(4):1518-1523.

19. Marchac V, Emond S, Mamou-Mani T, Le Bihan-Benjamin C, Le Bourgeois M, De Blic J, et al. Thoracic CT in pediatric subjects with difficult-to-treat asthma. AJR Am J Roentgenol 2002;179(5):1245-1252.

20. Homer RJ, Elias JA. Consequences of long-term inflammation. Airway remodeling. Clin Chest Med 2000;21(2):331-343.

21. Paganin F, Trussard V, Senéterre E, Chanez P, Giron J, Godard P, et al. Chest radiography and high resolution computed tomography of the lungs in asthma. Am Rev Respir Dis 1992;146(4):1084-1087.

22. Park JW, Hong YK, Kim CW, Kim DK, Choe KO, Hong CS. Highresolution computed tomography in subjects with bronchial asthma: correlation with clinical features, pulmonary functions and bronchial hyperresponsiveness. J Invest Allergol Clin Immunol 1997;7(3):186192.

23. Hudon C, Turcotte H, Laviolette M, Carrier G, Boulet LP. Characteristics of bronchial asthma with incomplete reversibility of airflow obstruction. Ann Allergy Asthma Immunol 1997;78(2):195-202.
24. Roche WR, Beasley R, Williams JH, Holgate ST. Subepithelial fibrosis in the bronchi of asthmatics. Lancet 1989;333(8637):520-524.

25. Chu HW, Halliday JL, Martin RJ, Leung DY, Szefler SJ, Wenzel SE. Collagen deposition in large airways may not differentiate severe asthma from milder forms of the disease. Am J Respir Crit Care Med 1998;158(6):1936-1944.

26. Matsuoka S, Uchiyama K, Shima H, Ueno N, Oish S, Nojiri Y. Bronchoarterial ratio and bronchial wall thickness on high-resolution CT in asymptomatic subjects: correlation with age and smoking. AJR Am J Roentgenol 2003;180(2):513-518.

27. Chetta A, Foresi A, Del Donno M, Bertorelli G, Pesci A, Olivieri D. Airways remodeling is a distinctive feature of asthma and is related to severity of disease. Chest 1997;111(4):852-857.

28. Safak AA, Arbak P, Yazici B, Bilgin C, Erdogmus B, Annakkaya AN, et al. Bronchial wall thickness in toll collectors. Ind Health 2010;48(3):317-323

29. Sarıman N, Levent E, Cubuk R, Yurtlu S, Benli Aksungar F. Bronchial hyperreactivity and airway wall thickening in obstructive sleep apnea patients. Sleep Breath 2011;15(3):341-350.

30. Haahtela T, Jarvinen M, Kava T, Kiviranta K, Koskinen S, Lehtonen $\mathrm{K}$, et al. The effects of reducing or discontinuing inhaled budesonide in subjects with mild asthma. N Engl J Med 1994;331(11): 700-705.

31. Selroos O, Pietinaloho O, Lofroos AB, Riska H. Effect of late versus early intervention with inhaled corticosteroids in asthma. Chest 1995; 108(5):1228-34.

32. Ernst P, FitzGerald JM, Spiers S. Canadian Asthma Guidelines: report from a consensus workshop. Can Respir J 1996;3:89-100.

33. Ward C, Pais M, Bish R, Reid D, Feltis B, Johns D, et al. Airway inflammation, basement membrane thickening and bronchial hyperresponsiveness in asthma. Thorax 2002;57(4):309-316. 